\title{
Functional Outcome of Patients with Lumbar Intervertebral Disc Herniation after Minimally Invasive Microdiscectomy
}

\author{
${ }^{* 1}$ Dr. Amlan Mohapatra, Senior Resident, \\ ${ }^{2}$ Dr. Bhaskar Bhandary, Associate Professor \\ ${ }^{3}$ Dr.Kartik Raj K, Junior Resident \\ ${ }^{123}$ Department of Orthopedics AJ Institute of Medical Sciences, Mangalore, Karnataka \\ *Corresponding author: *Dr. Bhaskar Bhandary, Associate Professor
}

\begin{abstract}
:
Introduction: Low back pain is very common worldwide and lumbosacral radiculopathy is an extremely painful condition. In this study we aimed to assess the functional outcome in patients who underwent minimally invasive microdisectomy at our institution.

Methodology: A prospective study was conducted in the Department of Orthopedics, AJ Institute of Medical Sciences, Mangalore, Karnataka from August 2014 till August 2016. After taking approval of the institutional ethics committee, all patients aged more than 18 years, who presented to the outpatient clinic of the Department of Orthopedics, AJ Institute of Medical Sciences, Mangalore, Karnataka with complaints of leg or back pain. We assesed pain and functionality using Japanese Orthopedic Association questionnaire (JOA) preoperatively, 1 month, 3 months and 6 months postoperatively. Patients were asked to rate their level of well being after surgery using the Macnab's criteria.

Results: We included 53 patients during the study period, average age was $40.17 \pm 8.5$ years. 32 patients were males. Using Macnab's criteria 35\% reported good level of well being and no patient reported poor level of well being at the end of 6 months postoperatively. $28.3 \%$ had excellent, $62.3 \%$ had good, $7.5 \%$ had fair and $1.9 \%$ had poor results with JOA score at the end of the study. JOA score improved from a mean of 10.3 preoperatively to 25.45 post-operatively at 6 months ( $p<0.001)$.

Conclusions: Findings of our study show that minimally invasive lumbar microdiscectomy cause very few postoperative complications, shorter stays in hospital, improve objective functionality scores postoperatively and patients rate well being assesment scores as good to excellent.
\end{abstract}

Keywords: microdisectomy, back pain, disc herniation, complications, pain

\section{Introduction}

Low back pain is very common worldwide and lumbosacral radiculopathy is an extremely painful condition. The cause is usually structural like disc herniation, leading to nerve root compression. Treatment for such patients with acute lumbosacral radiculopathy aims to decrease and improve upon the pain (symptomatic treatment) and to address the specific underlying process. When disc herniation causes radiculopathy symptomatic treatment is usually given during the acute period. However, mechanism-specific treatment is indicated if there is progressive neurologic compromise and/or persistent symptoms that are unresponsive to time and conservative measures. ${ }^{1}$ Nornarcotic analgesics, activity modification, physical therapy, opioid analgesics, glucocorticoids are some of the conservative management options avaliable for such patients. But a small percentage of patients require surgical interventions to alleviate their pain. There is no evidence that early referral for surgery, in the absence of severe or progressive neurologic deficits, improves outcomes for lumbar disc prolapse with radiculopathy or symptomatic spinal stenosis. ${ }^{2}$ There is a scarcity of literature from India which has reported on the success and postoperative assessments in patients who undergo microdisectomy for disc herniation. In this study we aimed to assess the functional outcome in patients who underwent minimally invasive microdisectomy at our institution. We used validated objective as well as subjective scales to assess the clinical outcomes in such patients.

\section{Study design and sample population}

\section{Methodology}

A prospective study was conducted in the Department of Orthopedics, AJ Institute of Medical Sciences, Mangalore, Karnataka from August 2014 till August 2016. A patient case reprort form was designed to collect patient realted informaton. Informed written consent was taken from all the patient prior to interviewing the patient. After taking approval of the institutional ethics committee, all patients aged more than 18 years, who presented to the outpatient clinic of the Department of Orthopedics, AJ Institute of Medical 
Sciences, Mangalore, Karnataka with complaints of leg or back pain, or other neurological symtoms, supported by Magnetic Resonance Imaging (MRI) suggestive of disc herniation, were included in the study. All patients were advised non-operative conservative measures first. Those patients who did not respond to conservative management were selected for minimally invasive microdisectomy. All patients included in the final sample population had a confirmed diagnosis of disc herniation and unilateral straight leg raise (SLR) test positive. We excluded patients aged more than 50 years because of their unsutaibility for undergoing a surgery procedure, those with asymptomatic or recurrent disc herniation, bilateral dischernation, history of lumbar spinal surgery, operative site infections, spinal infections, medically unfit for surgery, not willing for surgery or those with psychiatric illness. We also excluded patinets with back pain due to tumors, vertebral fractures, spinal canal stenosis, cauda equina or listhesis. All patients were followed for a period of 6 months postoperatively.

\section{Data collection and analysis}

Using the pre-designed semi-structured questionare we collected baic sociodemographic and clinical information of the patient. We assesed pre-operative and postoperative pain and functionality using Japanese Orthopedic Association questionnaire (JOA). ${ }^{3} \mathrm{JOA}$ was assessed for all patients preoperatively, 1 month, 3 months and 6 months postoperatively. All patients underwent minimally invasive microdisectomy. Patient was discharged once he/ she was pain free and able to ambulate on their own (usually on 5th post-operative day). Suture removal was performed after 10 days post-operative (after wound inspection). Patients were asked to rate their level of well being after surgery using the Macnab's criteria. ${ }^{4}$ This is a validated tool to assess patients' level of well being and satisfaction postoperatively. Macnab assessment was rated as $1=$ poor, $2=$ fair, $3=$ good, $4=$ excellent. Macnab's score was calculated at the time of surgery, 1 month, 3 months and 6 months postoperatively. Results after the surgery were assessed according to the rate of improvement, which was calculated using the following formula (Hirabayashi's method). ${ }^{5}$ Rate of Improvement (RI) = Postoperative score - Preoperative score / Total score (29) - Preoperative score] * 100. The rate of improvement (RI) was graded into 4 groups : Excellent as $\geq 90 \%$; Good as $75-89 \%$; Fair as $50-74 \%$ and Poor as $<49 \%$ improvement. ${ }^{6}$ Collected data was entered in Microsoft excel sheets and later imported in the Statistical Package for Social Sciences software for appropriate analysis. Significance over the period was calculated for JOA score by repeated measures ANOVA. For all statistical tests, pvalue $<0.05$ was considered significant and $p<0.001$ was highly significant. Statistical analysis was performed using SPSS 17 software (Statistical Package for Social Sciences).

\section{Results}

We included 53 patients during the study period, average age was $40.17 \pm 8.5$ years. 32 patients were males. Most of the patients have had symotoms for less than 6 months (Table 1). L4-L5 was the most commonly level involved. None of the patinets had any post-operative infections (Table 2). $40 \%$ of the patients had 5 to 6 days of hospital stay, followed by $32 \%$ having 7 to 10 days of hospital stay. We assessed the patients using the Macnab's criteria postoperatively at the time of surgery, 1 month, 3 months and 6 months after surgery. 57\% of the patients reported excellent level of well being at the end of 6 months as compared to only $4 \%$ at the time of surgery. 35\% reported good level of well being and no patient reported poor level of well being at the end of 6 months postoperatively (Table 2). JOA score was used to assess functionality and pain preoperatively and postoperatively. 15 cases $(28.3 \%)$ had excellent, 33 cases $(62.3 \%)$ had good, 4 cases $(7.5 \%)$ had fair and one $(1.9 \%)$ had poor results with JOA score at the end of the study. JOA score over the period was assessed by repeated measures ANOVA. JOA score improved from a mean of 10.3 pre-operatively to 25.45 post-operatively at 6 months (p value $<0.001$ ), with a rate of improvement $81.20 \%$ (Figure 1).

\section{Discussion}

Complications encountered with minimally invasive spine surgery are intraoperative bleeding, cerebrospinal fluid leak, nerve damage, wound infection, development of new neurological deficits and recurrence of disc herniation. In our study, our mean blood loss was $92.4 \mathrm{ml}$, accidental dural tear in one case and no infection rate correlating with Stolke's study, where incidental dural tears occurred in $1.8 \%$ of microdiscectomies. ${ }^{7}$ In a review by Ross $^{8}$ of 1231 cases operated over a 12 year period and in an earlier study by $\mathrm{O}^{\prime}$ Toole ${ }^{9}$ over 1338 cases operated by minimally invasive spinal surgery observed less rate of wound infection, durotomy and new neurologic deficits. The possible reasons hypothesised by the authors for the low rate of surgical site infection were reduced tissue exposure, minimal skin exposure to wound so less chance of contamination, smaller wounds heal rapidly, lack of use of monopolar coagulation, symmetrical distribution of retraction forces, incision made by single stroke and absence of skin sutures.

The success rates for standard discectomy range from $68 \%$ to $95 \%$, that of microdiscectomy from 88 $98.5 \%$ in different series and that of microendoscopic discectomy from 73-94\%. Of the 79 patients in Findlay et al study, 38 were men and 41 were women with a mean age of 38 years $(18-64)$ and average duration of 
symptoms 8.4 months ( 8 weeks to 36 months). ${ }^{10}$ All had single level lumbar disc herniations, with 44 cases at L4 - L5 and 39 at L5 - S1. They had a long term outcome of lumbar micro discectomy using Macnab criteria with an initial success rate of $91 \%$ at 6 months which declined only slightly to $83 \%$ at 10 -year follow-up. Our study findings are similar to these finding with a success rate of $92.4 \%$ at the end of our study in 6 months with Macnab criteria. Katayama et al in their study with 57 patients who underwent microdiscectomy, the mean age at surgery was 41 years, mean duration of follow-up was 2 years and 8 months and mean duration of hospitalization was $8.5 \pm 2.3$ days. ${ }^{11}$ The JOA scores improved from $16 \pm 2$ points before surgery to $27 \pm 1$ points for the last follow-up time, giving an improvement rate of $77 \%$. In comparison, at the end of our study, mean JOA score improved from 10.3 pre-operatively to 25.45 post-operatively at 6 months, giving a rate of improvement $81.2 \%$. Micro discectomy for symptomatic lumbar disc herniations in young, active patients has high success rates, especially with sequestered or extruded disc herniations than in those with contained disc herniations.

It has been considered by Wen et al that the choice of surgical method depends on the preference of doctors and personal ability. ${ }^{12} \mathrm{Lu}$ et al pointed out that it has been the goal to maintain lumbar stability under the premise of ensuring the efficacy with the orthopaedic surgeon damage to the lumbar spine of normal structure as little as possible. ${ }^{13}$ Though so many theoretical advantages of the minimally invasive lumbar discectomy compared to the conventional one are spoken off, they are yet to be proven for better patient outcomes ${ }^{14}$. Lumbar disc herniation removal techniques have evolved in terms of instrumentation without any appreciable improvement in clinical results. Correct indication, expertise of the surgeon and the patients informed consent remain the key factor in deciding the technique. ${ }^{15}$ A good control of the indication for surgery is the basis, accurate positioning is the prerequisite, protecting the nerve is the key, complete discectomy is the guarantee, full decompression is the fundamention and all the factors work together to make minimally invasive perfect. ${ }^{16}$

\section{Conclusion}

Microdiscectomy, as demonstrated by our outcome scores, has a high success rate for patients with lumbar disc herniations who have failed a period of conservative management. Findings of our study demonstrate that minimally invasive lumbar microdiscectomy cause very few postoperative complications, shorter stays in hospital, improve objective functionality scores postoperatively and patients rate well being assesment scores as good to excellent.

Table 1: Baseline socio-demographic characteristics of the patients included in the study

\begin{tabular}{|l|l|}
\hline & $n$ \\
\hline Number of patients & 53 \\
\hline Average age & $40.17 \pm 8.5$ years \\
\hline Males & 32 \\
\hline Duration of symptoms & \\
\hline 0 to 6 months & 28 \\
\hline 7 to 12 months & 20 \\
\hline More than 12 months & 5 \\
\hline Levels of lesions & \\
\hline L3-L4 & 7 \\
\hline L4-L5 & 36 \\
\hline L5-S1 & 10 \\
\hline
\end{tabular}

Table 2: Post-operative assessments of the patients

\begin{tabular}{|ll|}
\hline Variable & $\boldsymbol{n}(\boldsymbol{\%})$ \\
Post-operative infections & $53(100 \%)$ \\
Absent & $0(0 \%)$ \\
Present & \\
Length of stay in hospital (days) & \\
$3-4$ & $13(24 \%)$ \\
$5-6$ & $21(40 \%)$ \\
$7-10$ & $17(32 \%)$ \\
More than 10 days & $2(4 \%)$ \\
Postoperative Macnab assessment* & \\
Macnab score at the time of surgery & \\
1 & \\
2 & $7(13 \%)$ \\
3 & $14(26 \%)$ \\
4 & $30(57 \%)$ \\
Macnab score at 1 month after surgery & $2(4 \%)$ \\
1 & $2(4 \%)$ \\
2 & $14(26 \%)$ \\
\hline
\end{tabular}


Functional Outcome of Patients with Lumbar Intervertebral Disc Herniation after Minimally ...

\begin{tabular}{|ll|}
\hline 3 & $34(64 \%)$ \\
4 & $3(6 \%)$ \\
1 & \\
2 & $0(0 \%)$ \\
3 & $8(15 \%)$ \\
4 & $36(68 \%)$ \\
Macnab score at 3 months after surgery & $9(17 \%)$ \\
1 & \\
2 & $0(0 \%)$ \\
3 & $4(8 \%)$ \\
4 & $19(35 \%)$ \\
\hline
\end{tabular}

* Macnab assessment 1= Poor, 2= Fair, 3= Good, 4= Excellent

Table 3: Preoperative and postoperative assessment using Japanese Orthopedic Association score

\begin{tabular}{|c|c|c|c|c|}
\hline \multicolumn{2}{|l|}{ JOA Scores } & $n(\%)$ & Chi-Square & P value \\
\hline \multirow{4}{*}{ Preoperative } & Poor & $6(11.3 \%)$ & \multirow[t]{4}{*}{59.132} & \multirow{4}{*}{$<0.001$} \\
\hline & Fair & $44(83.0 \%)$ & & \\
\hline & Good & $3(5.7 \%)$ & & \\
\hline & Excellent & $0(0 \%)$ & & \\
\hline \multirow{4}{*}{ Postoperative at 1 month } & Poor & $2(3.8 \%)$ & \multirow[t]{4}{*}{35.811} & \multirow{4}{*}{$<0.001$} \\
\hline & Fair & $37(69.8 \%)$ & & \\
\hline & Good & $14(26.4 \%)$ & & \\
\hline & Excellent & $0(0 \%)$ & & \\
\hline \multirow{4}{*}{ Postoperative at 3 months } & Poor & $2(3.8 \%)$ & \multirow[t]{4}{*}{20.868} & \multirow{4}{*}{$<0.001$} \\
\hline & Fair & $26(49.1 \%)$ & & \\
\hline & Good & $25(47.2 \%)$ & & \\
\hline & Excellent & $0(0 \%)$ & & \\
\hline \multirow{4}{*}{ Postoperative at 6 months } & Poor & $1(1.9 \%)$ & \multirow[t]{4}{*}{47.453} & \multirow{4}{*}{$<0.001$} \\
\hline & Fair & $4(7.5 \%)$ & & \\
\hline & Good & $33(62.3 \%)$ & & \\
\hline & Excellent & $15(28.3 \%)$ & & \\
\hline
\end{tabular}

Figure 1: Mean rate of improvement in Japanese Orthopedic Assessment score preoperatively and postoperatively

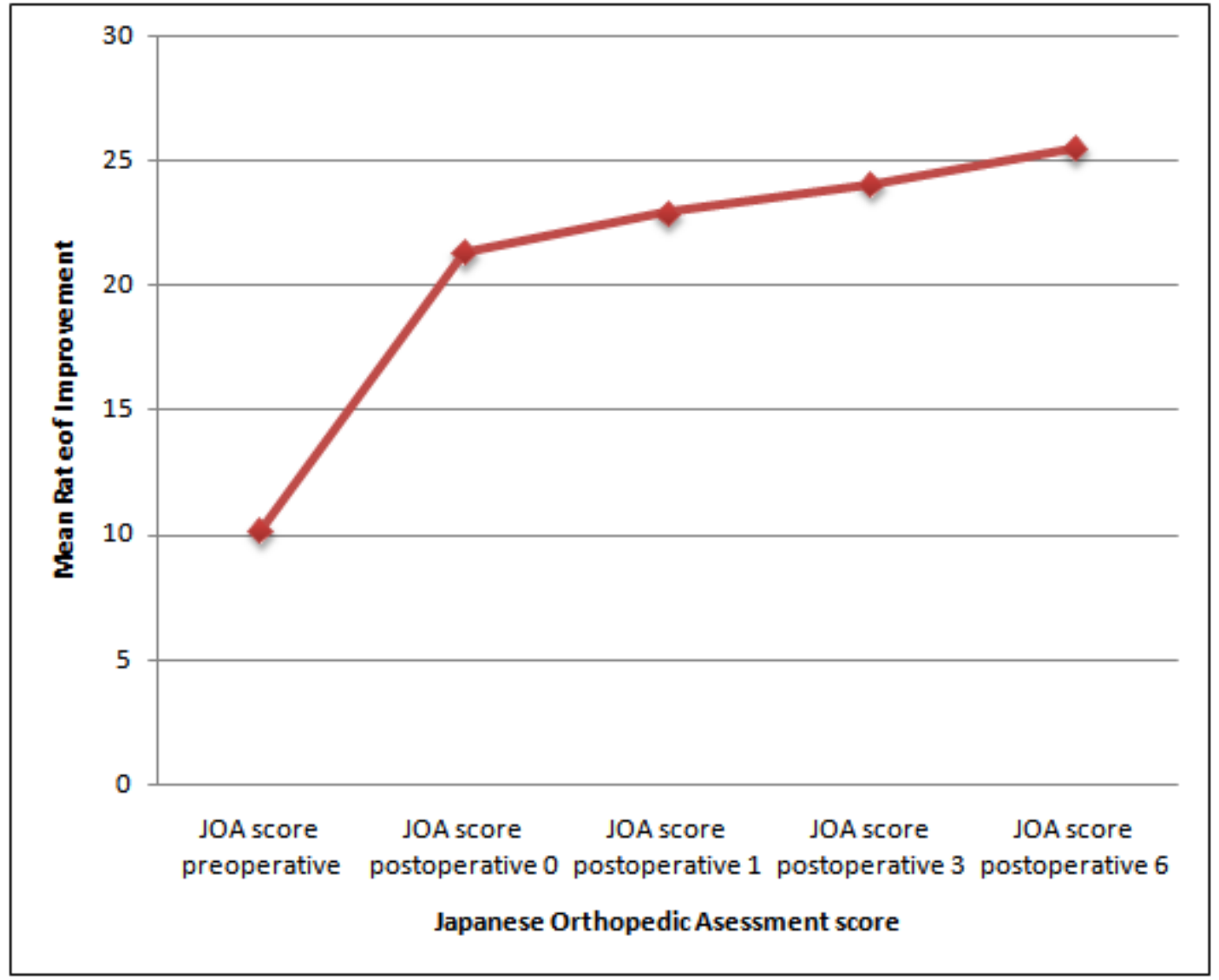

ANOVA F value $=1804.63, \mathrm{p}$ value $<0.001$, highly significant 


\section{References}

[1]. Pengel LH, Herbert RD, Maher CG, Refshauge KM. Acute low back pain: systematic review of its prognosis. BMJ 2003; $327: 323$.

[2]. Vroomen PC, de Krom MC, Knottnerus JA. Predicting the outcome of sciatica at short-term follow-up. Br J Gen Pract 2002; 52:119.

[3]. Fukui M, Chiba K, Kawakami M, Kikuchi SI, Konno SI, Miyamoto M, Seichi A, Shimamura T, Shirado O, Taguchi T, Takahashi K. Japanese orthopaedic association back pain evaluation questionnaire. Part 3. Validity study and establishment of the measurement scale. Journal of Orthopaedic Science. 2008 May 1;13(3):173.

[4]. Macnab IA. Negative disc exploration. J Bone Joint Surg Am. 1971 Jul 1;53(5):891-903.

[5]. Hirabayashi K, Miyakawa J, Satomi K, Maruyama T, Wakano K. Operative results and postoperative progression of ossification among patients with ossification of cervical posterior longitudinal ligament. Spine. 1981 Jul 1;6(4):354-64

[6]. Chen Z, Zhao J, Liu A, Yuan J, Li Z. Surgical treatment of recurrent lumbar disc herniation by transforaminal lumbar interbody fusion. International orthopaedics. 2009 Feb 1;33(1):197-201.

[7]. Stolke D, Sollmann WP, Seifert V. Intra-and postoperative complications in lumbar disc surgery. Spine. 1989 Jan 1;14(1):56-9.

[8]. Ross DA. Complications of minimally invasive, tubular access surgery for cervical, thoracic, and lumbar surgery. Minimally invasive surgery. $2014 \mathrm{Jul}$ 7;2014.

[9]. O'Toole JE, Eichholz KM, Fessler RG. Surgical site infection rates after minimally invasive spinal surgery: clinical article. Journal of Neurosurgery: Spine. 2009 Oct;11(4):471-6.

[10]. Findlay GF, Hall BI, Musa BS, Oliveira MD, Fear SC. A 10- Year Follow- Up of the Outcome of Lumbar Microdiscectomy. Spine. 1998 May 15;23(10):1168-71.

[11]. Katayama Y, Matsuyama Y, Yoshihara H, Sakai Y, Nakamura H, Nakashima S, Ito Z, Ishiguro N. Comparison of surgical outcomes between macro discectomy and micro discectomy for lumbar disc herniation: a prospective randomized study with surgery performed by the same spine surgeon. Clinical Spine Surgery. 2006 Jul 1;19(5):344-7.

[12]. Wen SF, Liu EZ, Guo DM, Cai WS, Zhong BF, Yan H, Chen SM, Xu ZH. Comparison of surgical outcomes between microsurgery lumbar discectomy and microendoscopic discectomy for lumbar disc herniation. Chinese Journal of Microsurgery. 2008;31:104-6.

[13]. Lu X, Li W, He J, Peng X, Zhao J. A Retrospective Study of Microscope-Assisted Discectomy versus Open Fenestration Discectomy for Lumbar Disc Herniation. Annals of Clinical and Laboratory Research. 2016.

[14]. Majeed SA, Vikraman CS, Mathew V, Anish TS. Comparison of outcomes between conventional lumbar fenestration discectomy and minimally invasive lumbar discectomy: an observational study with a minimum 2-year follow-up. Journal of orthopaedic surgery and research. 2013 Sep 24;8(1):34.

[15]. Blamoutier A. Surgical discectomy for lumbar disc herniation: surgical techniques. Orthopaedics \& Traumatology: Surgery \& Research. 2013 Feb 28;99(1):S187-96.

[16]. Lu X, Li W, He J, Peng X, Zhao J. A Retrospective Study of Microscope-Assisted Discectomy versus Open Fenestration Discectomy for Lumbar Disc Herniation. Annals of Clinical and Laboratory Research. 2016.

Dr. Amlan Mohapatra. "Functional Outcome of Patients with Lumbar Intervertebral Disc Herniation after Minimally Invasive Microdiscectomy." IOSR Journal of Dental and Medical Sciences (IOSR-JDMS) 16.7 (2017): 35-39. Web. 